\title{
Tuning charge storage properties of supercapacitive electrodes evi- denced by in situ gravimetric and viscoelastic explorations
}

\author{
Wanli Gao', Catherine Debiemme-Chouvy', Mohammed Lahcini ${ }^{2,3}$, Hubert Perrot ${ }^{1, *}$ Ozlem Sel $^{1, *}$
}

\author{
'Sorbonne Université, CNRS, Laboratoire Interfaces et Systèmes Electrochimiques, LISE, 75005 Paris, France. \\ ${ }^{2}$ Cadi Ayyad Université, Faculté des Sciences et Techniques, Laboratoire Chimie Organométallique et Macromolé- \\ culaire -Matériaux Composites - Marrakech, Morocco. \\ 3Mohammed VI Polytechnic University, UM6P, 43150 Ben Guerir, Morocco.
}

\begin{abstract}
Revealed by an integrated electrogravimetric and viscoelastic method, slightly electrochemically reduced graphene oxide (ERGO) presents an anion preference for charge storage and delivery, while with the progressive removal of oxygen functionalities on its basal planes, cations begin to predominate in charge compensation. This "anion-to-cation" evolution in neutral aqueous media can not only affect the electrochemical charge storage, but also play an important role in electrode's viscoelasticity. It was demonstrated that oxygen functionalities could modify the interactions between graphene layers and even contribute to pseudocapacitances. However, the role of oxygen functionalities in species transfer and viscoelastic variations still remains poorly understood. Herein, a combined methodology of electrochemical quartz crystal microbalance (EQCM), ac-electrogravimetry and electroacoustic impedance measurements was proposed for characterizing the electrochemical and viscoelastic responses of graphene oxides with various degree of electrochemical reduction. With the removal of oxygen containing functional groups, ERGO electrode exhibits (i) a gradually enhanced specific capacitance $(C s)$ with increased flexibility (decreased storage moduli, $G^{\prime}$ ); (ii) a dehydration process of cations (i.e. from $\mathrm{Na}^{+} .2 \mathrm{H}_{2} \mathrm{O}$ to $\mathrm{Na}^{+} . \mathrm{H}_{2} \mathrm{O}$ ); and (iii) a potential-dependent "stiffened-softened" behavior. These results open the door for a suitable design of GO-based materials for electrochemical energy storage and shed light on electronic devices where ion-selective behavior plays a key role.
\end{abstract}

Owing to high power density and superior cycling performance, supercapacitor (SC) has boosted numerous research efforts in electrochemical energy storage and offered a wide range of potential applications, such as portable electronics, electric vehicles and stand-free power systems $^{1-6}$. The most important component in SC is the electrode material with high surface area and high porosity ${ }^{7-8}$. Among those, carbon-based materials are considered as prospective SC electrode candidates for bulk production ${ }^{9-}$ ${ }^{10}$. Graphene, a sp2-bonded carbon monolayer, has demonstrated itself significantly effective for electrochemical energy storage/delivery due to many appealing features, such as high surface area (theoretically as high as $2630 \mathrm{~m}^{2} \cdot \mathrm{g}^{-1}$ ), superior mechanical properties, excellent electronic conductivity and chemical resilience ${ }^{2,10-15}$. Reduction of graphene oxide (GO) is widely adopted for the production of graphene because of its low cost, high scalability and competitive yield ${ }^{16-17}$. As a highly defective form of graphene functionalized by enriched oxygen-containing groups, GO may offer higher capacitance than graphene through additional pseudo-capacitive behavior ${ }^{18-21}$. However, the oxygen containing functional groups can also restrain the sp2 hybridization and thus render the GO less conductive due to degraded $\pi-\pi$ stacking. From this perspective, the electrochemical charge storage performance of GO is strongly associated with the content of oxygen functional groups on its layers: $i$ ) high content of oxygen functionalities brings about more pseudocapacitance from reversible redox reactions and improves the wettability of GO electrodes with aqueous solution but results in an inferior conductivity and less electroactive surfaces for electrical double-layer capacitance (EDLC); contrarily, ii) low content of oxygen functional groups leads to an enhanced electronic conductivity due to the restoration of the $\pi-\pi$ network and a higher electroactivity of the surfaces for charge adsorption/desorption, thereby promoting improvements in electrochemical performances of SC. Thus, a direct correlation between the oxygen-containing groups of the GO and the final electrochemical performance of the SCs cannot be disregarded.

Under the consideration that most of the graphenes synthesized through reduction of GO bear some unavoidable extrinsic defects from oxygen-containing groups ${ }^{16,22}$, a systematic investigation on the electrochemical behavior of GO with different content of oxygen functionalities is highly desirable for well-designed SC electrodes. Additionally, although it has been demonstrated that oxygen 

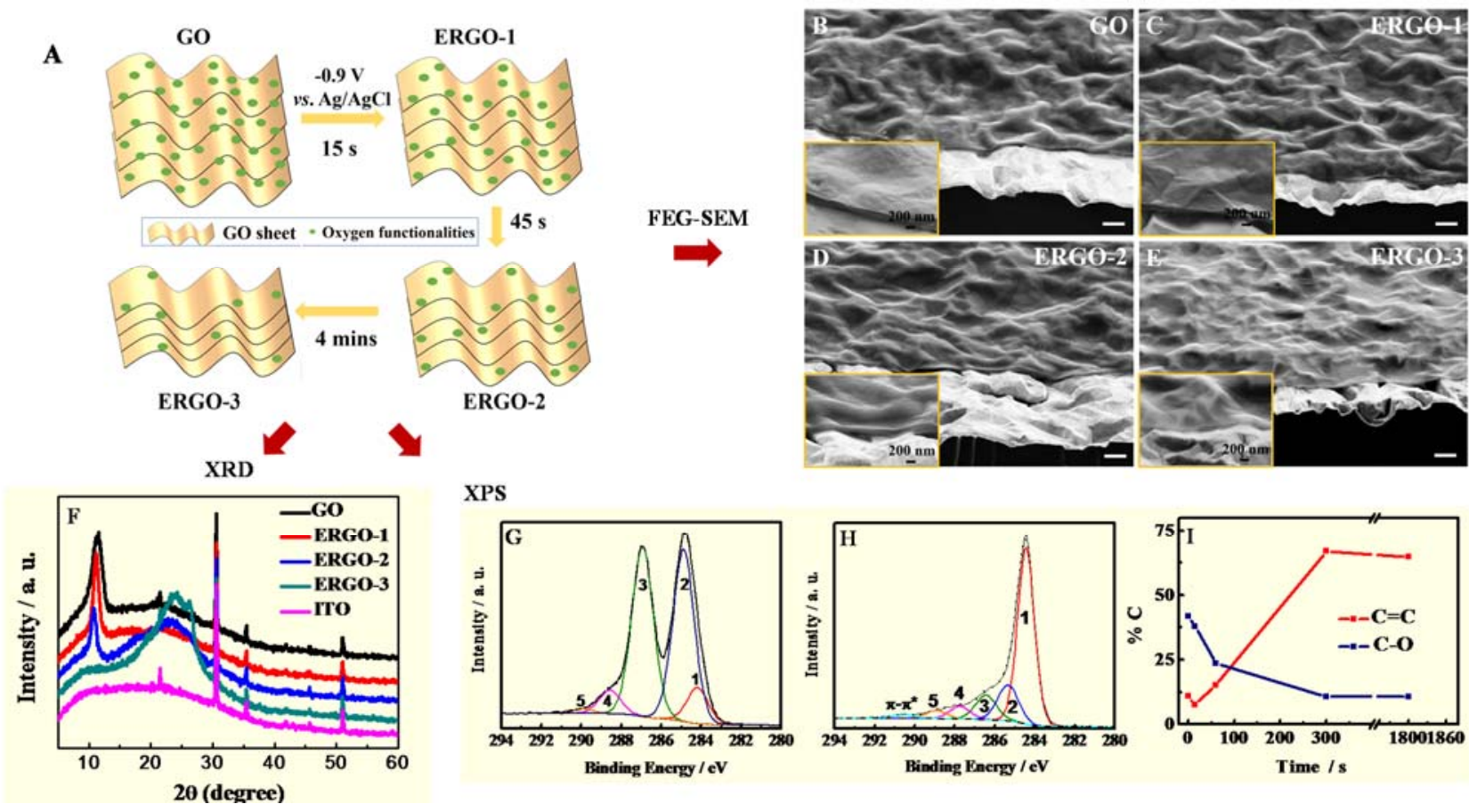

XPS
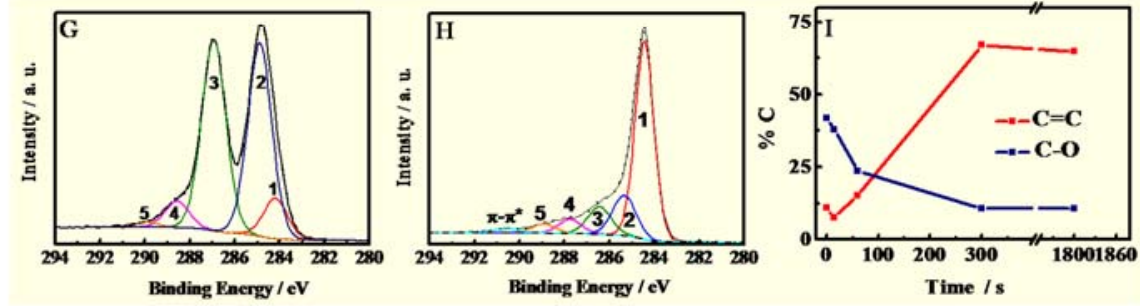

Figure 1. (A) Synthesis process and the expected structural changes for ERGO-1, -2 and -3; morphological observations of graphene oxide film (B) and GO films with different degree of reduction, ERGO-1 to ERGO-3 (C-E) (white scale bars: $1 \mu \mathrm{m}$ ). Inset images are higher magnification of each film; (F) XRD patterns of pristine GO and GO with various degree of electrochemical reduction and XPS spectra of GO $(\mathrm{G})$ and ERGO-3 $(\mathrm{H})$, where the curves were fitted considering the following contributions: $\mathrm{C}=\mathrm{C}$ ( $\mathrm{sp2}$; peak 1), C-C (sp3; peak 2), C-O (epoxy and hydroxyl; peak 3), C=O (carbonyl; peak 4), O-C=O (carbonyl; peak 5) and $\pi-\pi^{*}$. The panel (I) presents the relative percentage of $\mathrm{C}=\mathrm{C}$ and $\mathrm{C}-\mathrm{O}$ groups as a function of time (The lines are a guide to eyes).

containing functional groups could bring about reversible electrochemical reactions, thus contributing to capacitance ${ }^{18-19}$, the mechanism of ionic fluxes during charge-discharge process in carbon based electrodes still remains poorly understood.

Besides superior electrochemical properties, long-term cycling performances are also of importance for an efficient SC electrode. Upon cycling, periodical ion electroadsorption/electrodesorption and/or insertion/extraction are possibly accompanied with solvent flux which lead to repeated species-transfer-induced deformation as well as viscoelastic changes of the GO electrode (stiffened/softened). Such viscoelastic changes which are not completely relaxed at the end of each cycle and tend to gradually accumulate, thereby result in a failure of the mechanical integrity and lead to an eventual cyclability fading. Multiharmonic EQCM with dissipation monitoring (EQCM-D) has been used for the investigation of gravimetric and viscoelastic changes of electrodes during charge storage process by the interpretation of both frequency $(\Delta f / n, n$ is the overtone order) and resonance width $(\Delta W / n)$ changes $^{23}$. Nevertheless, of practical and fundamental importance but still scarcely touched is the correlation between electrode viscoelasticity and its electrochemical performance ${ }^{24-26}$.
Electrochemical quartz-crystal microbalance (EQCM) and its coupling with electrochemical impedance spectroscopy (the so-called ac-electrogravimetry) have proved themselves significantly effective for evaluating the electrochemical behaviors of carbon-based electrodes ${ }^{27-28}$, particularly in situ capturing ionic transfer between the electrode and electrolyte. Besides identifying each species participating in the charge compensation process, it can provide a quantitative picture together with the kinetics of transfer of each species, thereby providing a kinetic and gravimetric deconvolution. In addition to the utility of the electrogravimetric methods, the viscoelastic evolutions of the GO electrodes in the removal process of oxygen-containing groups and at different states-of-polarization can be tracked by electroacoustic impedance method ${ }^{29-31}$, from which two important parameters for describing the viscoelasticity of the GO electrodes, i.e., the storage $\left(G^{\prime}\right)$ and loss moduli $\left(G^{\prime \prime}\right)$ can be estimated. $G^{\prime}$ represents the electrode stiffness whereas $G^{\prime \prime}$ reflects its viscosity. These parameters can be further utilized to estimate the sensitivity coefficients of the QCM when it is not under gravimetric regime and thus help to differentiate the effect of the mechanical property changes from purely gravimetric responses $^{29}$.

In the current study, ERGO electrodes with different content of oxygen functionalities were prepared and a combined methodology of EQCM, ac-electrogravimetry 
and electroacoustic impedance method was proposed to acquire a deeper insight into (i) the influence of oxygen containing functional groups on the ionic transfer in ERGO electrodes and (ii) the correlation between electrochemical and viscoelastic evolution during the gradual removal of these groups. To the best of our knowledge, such a systematic study is unprecedented and is distinguished from the previous works thanks to the non-conventional electrochemical characterization methods employed, which provides the correlation between oxygen containing functional groups and nanostructure of the ERGO layers and their effect on the species (ions or solvent) transfer kinetics, hydration/dehydration of ions and the electrochemical and viscoelastic properties of the electrodes.

\section{EXPERIMENTAL SECTION}

Synthesis of ERGO electrodes and structural characterization. GO was synthesized by a modified Hummers method $^{32}$. GO suspension of $1 \mathrm{mg} / \mathrm{mL}$ was prepared from addition of GO powder to bi-distilled water, followed by a consecutive ultrasonication for $5 \mathrm{~h}$ to get a homogeneous solution. About $40 \mu \mathrm{L}$ GO suspension were deposited on the gold electrode $\left(0.2 \mathrm{~cm}^{2}\right)$ of a quartz crystal microbalance (QCM) resonator (9 MHz-AWS, Valencia, Spain) with the help of a lab-made mask, which ensures the exposure of the gold electrode only. GO film was generated after being dried at $70^{\circ} \mathrm{C}$ for $1 \mathrm{~h}$ in an oven. The electrochemical reduction was carried out by a standard three electrode configuration, where GO-coated gold electrode of QCM was used as a working electrode, with a platinum grid and $\mathrm{Ag} / \mathrm{AgCl}$ ( $3 \mathrm{M} \mathrm{KCl}$ saturated with $\mathrm{AgCl}$ ) as a counter and a reference electrode, respectively. The reduced GO films were electrochemically synthetized by chronoamperometry with a Biologic SP-20o potentiostat using EC-Lab software at $-0.9 \mathrm{~V}$ vs. $\mathrm{Ag} / \mathrm{AgCl}$ in a solution of $0.5 \mathrm{M} \mathrm{NaNO}_{3}$. Nitrogen gas was bubbled in the solution prior to the electrochemical reduction and was maintained over the solution until the end of the reduction to ensure a complete saturation in $\mathrm{NaNO}_{3}$ solution. The resulting ERGO electrodes were designated as ERGO-1, ERGO-2 and ERGO-3 in accordance with the reduction time of $15 \mathrm{~s}, 1 \mathrm{~min}$ and $5 \mathrm{~min}$, respectively (Figure $\mathbf{1 A}$ ).

The morphologies of the electrogenerated ERGO electrodes were observed under vacuum conditions using a field emission gun scanning electron microscope (FEGSEM, Zeiss, Supra 55). The crystallographic structures were determined by X-ray diffraction (XRD), which was performed using Philips PANalytical X'Pert Pro diffractometer with $\mathrm{Cu} \mathrm{K} \alpha$ radiation $(\lambda=1.54184 \AA)$. The electrodes used for XRD were prepared on ITO-coated glass slides and reduced under the same conditions as those on gold electrode of the QCM. X-ray photoelectron spectroscopy analyses were performed using an Omicron Argus X-ray photoelectron spectrometer with monochromatized $\mathrm{Al} \mathrm{K \alpha}$ excitation $(1486.6 \mathrm{eV})$ with a pass energy of $100 \mathrm{eV}$ and $20 \mathrm{eV}$
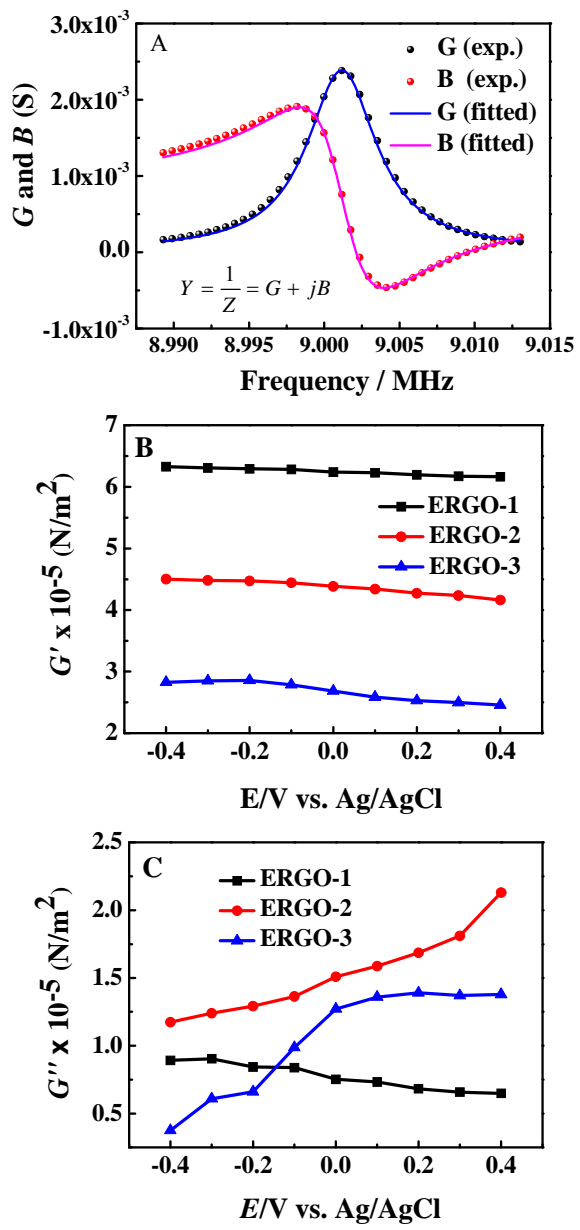

Figure 2. (A) Electroacoustic admittance measurements: experimental and fitted conductance and susceptance over frequency for ERGO-1 at -0.2 V. Fitting parameters: $G^{\prime}=6.29 \times 10^{5}$ N.m ${ }^{-2}, G^{\prime \prime}=0.84 \times 10^{5} \mathrm{~N} . \mathrm{m}^{-2}, d_{f}=146 \mathrm{~nm}$ and $\rho_{f}=2 \mathrm{~g} . \mathrm{cm}^{-3}$; evolution of real (B) and imaginary $(\mathrm{C})$ components of complex shear modulus of ERGO electrodes.

for acquisition of the survey and high-resolution spectra, respectively. Further details are given in the Supporting Information.

Electroacoustic impedance measurements. The viscoelastic evolutions were explored through the electroacoustic impedance measurements to elucidate the relationship between electrochemical and viscoelastic changes of the ERGO films 33,34 . They were carried out in the potential range between $-0.4 \mathrm{~V}$ and $0.4 \mathrm{~V}$ (at nine different potentials with an interval of $100 \mathrm{mV}$ ). An Agilent 4294A impedance analyzer coupled with a lab-made potentiostat was used to perform the electroacoustic impedance measurements under polarization. A lab-made software Simad was used for extracting storage moduli $\left(G^{\prime}\right)$ and loss moduli $\left(G^{\prime \prime}\right)$ of the ERGO films through fitting the experimental data. $G^{\prime}$ is associated with the energy storage capability of the ERGO layers whereas $G^{\prime \prime}$ describes the energy dissipation of the electrode due to internal friction and irreversible deformation of ERGO sheets. 
The complete theoretical electrical admittance, $Y_{t h}^{V}(\omega)$, of the loaded quartz resonator is given in Eq.1:

$$
Y_{t h}^{V}(\omega)=\frac{1}{Z_{t h}^{V}(\omega)}=G+i B=i \times \omega \times C_{p}+\frac{1}{Z_{m}^{V}(\omega)}
$$

where $G$ is the conductance (which can be written as a complex number with a real and imaginary part, $G=G+j G^{\prime \prime}$, where $\left.j^{2}=-1\right), B$ depicts the susceptance, $\omega=2 \times \pi \times f, C_{p}$ is the parasitic capacitance and $Z_{m}^{V}$ is the motional impedance of the loaded quartz resonator calculated with a viscoelastic model (see Supporting Information for details of the data treatment based on the work of Martin et al33). Figure 2A shows an example for the fitting of experimental response of ERGO-1 film at -0.2 V, which takes into consideration four parameters of the film, i.e., film thickness $(d$,

), film density $(\rho),, G^{\prime}$ and $G^{\prime \prime}$.

The methodology used to treat the viscoelastic properties of ERGO layers is based on the electroacoustic measurements and the data treatment by Martin's model, which is itself based on the transmission line modelling (TLM) ${ }^{33}$. This study is complementary to the recent reports on the viscoelastic and gravimetric behaviors of $2 \mathrm{D} \mathrm{Ti}_{3} \mathrm{C}_{2}$ (MXene) electrodes by multiharmonic EQCM-D where a Voigt-type modelling has been used ${ }^{23,24,26}$. Therefore, a brief discussion on both models (Voigt type 35 and TLM) are given in the Supporting Information.

Electrogravimetric measurements. The electrogravimetric response of a pristine GO thin film from ac-electrogravimetry ( $A C$-EG) was too weak to be analyzed due to its poor electronic conductivity. Therefore, the pristine GO film was slightly reduced for $15 \mathrm{~s}$ at $-0.9 \mathrm{~V}$ vs. $\mathrm{Ag} / \mathrm{AgCl}$ (ERGO-1) with the expectation of maintaining as much oxygen containing functional groups as possible on its sheets, and meanwhile being sufficiently conductive for obtaining a cognitive response in $A C$-EG. The electrochemical performances of ERGO electrodes were measured employing a standard three-electrode electrochemical cell which consisted of a ERGO-loaded quartz resonator as the working, $\mathrm{Ag} / \mathrm{AgCl}$ as the reference, and platinum grid as the counter electrode. Electrochemical experiments were carried out at ambient temperature in $1 \mathrm{M} \mathrm{NaCl}$. The potential window for galvanostatic charging-discharging (GCD) tests at different current densities $\left(0.5,1\right.$ and $\left.2 \mathrm{~mA} \cdot \mathrm{cm}^{-2}\right)$ was confined between $-0.5 \mathrm{~V}$ and $0.5 \mathrm{~V}$ vs. $\mathrm{Ag} / \mathrm{AgCl}$, which was applied to cyclic voltammetry $(\mathrm{CV})$ measurements as well. Both GCD and CV were coupled with the QCM measurements to obtain the simultaneous frequency variations of the modified quartz resonators.

$A C$-EG which couples the electrochemical impedance spectroscopy with fast QCM measurements were detailed else where ${ }^{27-28}$. Briefly, a four-channel frequency response analyzer (FRA, Solartron 1254) and a lab-made potentiostat were used for $A C$-EG. The QCM was performed under dynamic regime, and the modified working electrode was polarized at selected potentials. The frequency range for this potential perturbation was between $63 \mathrm{kHz}$ and $10 \mathrm{mHz}$. The microbalance frequency shift $\left(\Delta f_{m}\right)$ corresponding to the mass change $(\Delta m)$ of the modified working electrode was measured simultaneously with the current response $(\Delta I)$ or the charge response $(\Delta q)$ of the electrochemical system. The resulting signals were sent to a FRA, which allowed the electrochemical impedance $\left(\frac{\Delta E}{\Delta I}(\omega)\right)$, the electrogravimetric transfer function (TF) $\left(\frac{\Delta m}{\Delta E}(\omega)\right)$ and the charge/potential TF $\left(\frac{\Delta q}{\Delta E}(\omega)\right.$ to be obtained simultaneously at a given potential and frequency modulation, $f$ (pulsation $\omega=2 \pi f$ ) (see theoretical part in the Supporting Information).

\section{RESULTS AND DISCUSSION}

Morphology and structure of ERGO electrodes. ERGO thin films with progressively decreased content of oxygen functionalities were electrochemically fabricated as illustrated in Figure 1A. With increased reduction time, the ERGO sheets gradually lost the oxygen functional groups which was accompanied by a decrease of interspacing between the layers. The morphology of the GO and ERGO electrodes was observed by FEG-SEM as shown in Figure 1B-E. Both GO and ERGO electrodes displayed a typical wrinkled morphology, which is formed by thin crumpled sheets.

Sauerbrey equation was employed to calculate the film thickness in air through the relationship between mass change $(\Delta m)$ of the ERGO electrode and microbalance frequency shift $(\Delta f)$ before and after the electrochemical reduction of the film ${ }^{36}$, i.e., $\Delta f=-C_{f} \times \Delta m$, where $C_{f}$ is the modified sensitivity factor of the quartz crystal resonator $\left(C_{f}\right.$ $=19.5 \times 10^{7}, 18.7 \times 10^{7}$ and $\sim 17.8 \times 10^{7} \mathrm{~Hz} \cdot \mathrm{g}^{-1} . \mathrm{cm}^{2}$ for ERGO-1, -2 and -3 , respectively. See details in the viscoelastic properties section). The estimated thickness from Sauerbrey equation is $\sim 146, \sim 122$ and $\sim 100 \mathrm{~nm}$ for ERGO-1, -2 and -3 , respectively. These values were used for the fitting of the electroacoustic and electrogravimetric data thereafter.

The structural difference of GO electrodes before and after electrochemical reduction was characterized with XRD measurements. Figure $\mathbf{1 F}$ shows the XRD patterns of the pristine GO on ITO coated glass slides, revealing a sharp and intense diffraction peak centered at $2 \theta=11.5^{\circ}($ ooz $)$, corresponding to an interplanar spacing (d-spacing) of 0.77 $\mathrm{nm}$. This value is dependent on the preparation method and the number of water molecules between the layers ${ }^{37}$. After $15 \mathrm{~s}$ of electrochemical reduction, ERGO-1 displayed almost the same reflection peak, confirming that ERGO-1 shared almost the same structural geometry as GO in spite of a measurable thickness decrease observed by FEG-SEM and weight loss by QCM. It may be attributed to the superficial oxygen functional groups on GO layers and water molecules located in the shallow sites between GO layers, which can be easily removed during electrochemical reduction process. However, in the case of ERGO-2, the intensity of the peak (002) drastically faded and a new, broad 
peak emerged at $2 \theta=23.5^{\circ}$ corresponding to a d-spacing of $0.38 \mathrm{~nm}$. The decreased value of d-spacing in ERGO-2 implied that a significant portion of oxygen functional groups had been removed from GO sheets. Moreover, in the pattern of ERGO-3, the peak at $2 \theta=11.5^{\circ}$ disappeared and was markedly shifted to $23.5^{\circ}$, indicating that at least a partial reduction of GO was achieved.

The reduction degree of GO electrodes is further investigated by XPS. C1s spectrum of GO electrode (Figure 1G) displays its two main components of C-O (epoxy and hydroxyl, $\sim 286.9 \mathrm{eV})$ and $\mathrm{C}=\mathrm{C} / \mathrm{C}-\mathrm{C}(\sim 284.8 \mathrm{eV})$ in aromatic rings, and two minor components i.e., $\mathrm{C}=\mathrm{O}$ (carbonyl, $\sim 288.6 \mathrm{eV}$ ) and $\mathrm{O}-\mathrm{C}=\mathrm{O}$ (carboxyl, $\sim 290.0 \mathrm{eV}$ ) groups ${ }^{38-41}$. After electrochemical reduction for $5 \mathrm{~min}$, all intensities of the oxygen functionalities remarkably decrease (Figure $\mathbf{1 H})$, especially $\mathrm{C}-\mathrm{O}$ groups, evidencing that the majority of oxygen functionalities have been successfully removed. Furthermore, a stronger $\pi-\pi^{*}$ shake-up satellite peak around $290.7 \mathrm{eV}$ resumes in ERGO-3, which is a characteristic of aromatic or conjugated systems ${ }^{42}$. Figure 1I presents the variation of the two major groups $(\mathrm{C}=\mathrm{C}$ and $\mathrm{C}-\mathrm{O})$ as a function of reduction time. It clearly shows that the main oxygen functional group $(\mathrm{C}-\mathrm{O})$ is gradually removed upon electrochemical reduction and ERGO electrode can be sufficiently reduced after $5 \mathrm{~min}$, which is also confirmed by the $\mathrm{C} / \mathrm{O}$ ratio in Figure SI-1. These results, combined with XRD analysis, indicate the formation of ERGO with different content of oxygen functionalities.

Viscoelasticity of ERGO electrodes and its influence on electrogravimetric performance. Long term mechanical integrity is of significant importance for a high performance SC electrode. However, it is known that the periodical exchange of the species between the electrode and the electrolyte commonly results in the accumulation of unrelaxed structural deformations thus leading to the mechanical failure. Furthermore, it has been demonstrated that the viscoelastic properties of the deposited film play an essential role in the validity of Sauerbrey equation and can modify the gravimetric response of a quartz resonator $^{29}$. Therefore, when QCM based methodologies are employed to study the electroadsorption or insertion behavior, special attention should be given to distinguish between the (electro)gravimetric and the (electro)mechanical property changes of the electrodes. Hence, in this study, prior to the electrogravimetric investigations, electroacoustic impedance tests (Figure 2) were carried out to explore the influence of the progressive removal of oxygen functionalities from ERGO sheets on its viscoelastic evolution, which is described by the storage modulus $\left(G^{\prime}\right)$ and the loss modulus $\left(G^{\prime \prime}\right)$. As shown in Figure 2B, ERGO layers exhibit a decrease in the rigidity with progressive removal of oxygen containing functional groups from ERGO-1 to -3. For instance, $G^{\prime}$ of the ERGO-1 is reduced by approximately $58 \%$ from $\sim 6.2 \times 10^{5} \mathrm{~N} \cdot \mathrm{m}^{-2}$ to $\sim 2.6 \times 105 \mathrm{~N} \cdot \mathrm{m}^{-2}$ for ERGO-3, which are comparable to that of graphene hydro- gel ${ }^{43}$ and that of a composite $\mathrm{LiFePO}_{4}$ electrode used in batteries $^{44}$. By contrast, $G^{\prime \prime}$ shows a more complex evolution behavior (Figure $\mathbf{2 C}$ ). Specifically, $G^{\prime \prime}$ of ERGO-1 remains at lower values at most potentials and decreases gradually from cathodic to anodic potentials (from $0.89 \times 10^{5} \mathrm{~N} \cdot \mathrm{m}^{-2}$ to $0.65 \times 10^{5} \mathrm{~N} \cdot \mathrm{m}^{-2}$ ). However, in the case of ERGO-2 and $-3, G^{\prime \prime}$ exhibits an entirely opposite variation tendency with respect to ERGO-1, increasing from $1.17 \times 10^{5} \mathrm{~N} \cdot \mathrm{m}^{-2}$ to $2.13 \times 10^{5}$ $\mathrm{N} \cdot \mathrm{m}^{-2}$ and $0.37 \times 10^{5} \mathrm{~N} \cdot \mathrm{m}^{-2}$ to $1.38 \times 10^{5} \mathrm{~N} \cdot \mathrm{m}^{-2}$, respectively.

It has been revealed that the $G^{\prime}$ describes the film rigidity (energy storage), whereas $G^{\prime \prime}$ is correlated with film viscosity (energy dissipation) ${ }^{45}$. Although the measurements of the complex shear modulus are macroscopic and the electrochemical/electroacoustic measurements are not necessarily structural probes, a plausible molecular level interpretation of these macroscopic observations are as follows: The decreased $G^{\prime}$ and increased $G^{\prime \prime}$ from ERGO-1 to -2 reveal that the removal of oxygen containing functional groups and the corresponding restoration of the $\pi$ network endow the reduced graphene oxide (RGO) layers with less rigidity and more viscosity. It is speculated that strong polar interactions exist between the reduced graphene oxide interlayers mainly from enriched oxygen functionalities in ERGO-1 electrode, resulting in an inferior electrode conductivity and thus poor ions' transfer between electrode/electrolyte interface and transport in electrode bulk. Correspondingly, the friction between the ions and oxygen containing functional groups is greatly weakened and the relative displacement between ERGO layers hardly occurs, through which the energy dissipates during electrochemical process. With the removal of oxygen functionalities from ERGO-1 to -2, such strong polar interactions can be gradually replaced by restored $\pi$ system, which brings about the exchange of ions in a higher quantity between the electrode and electrolyte. Also, the corresponding deformation of ERGO sheets can be compatible for the accommodation to these increased amount of ions under different states-of-polarization. It in turn enhances energy dissipation in this process by means of $i$ ) enhanced friction between exchanged species and ERGO layers and ii) relative displacement between ERGO layers. Such properties can be presented by high flexibility and high viscosity, i.e., low $G^{\prime}$ and high $G^{\prime \prime}$.

In contrast with ERGO-2, ERGO-3 displays a further decreased $G^{\prime}$ due to the further removal of hetero-oxygen groups, however, a smaller $G^{\prime \prime}$ instead, implying that the graphene oxide sheets with higher degree of reduction become more flexible and less viscous. As a matter of fact, the gradual removal of oxygen containing functional groups (from ERGO-2 to ERGO-3) results in a further approach of the adjacent layers (the $d$-spacing of $0.38 \mathrm{~nm}$ was achieved in ERGO-3 as shown by XRD, Figure $\mathbf{1 F}$ ) and leads to the further restoration of its $\pi$ network with much larger conjugated length as compared to ERGO-2, which may weaken the internal friction between ERGO layers and thus attenuate the dissipation (lower $G^{\prime \prime}$ ). Besides, it may facilitate 

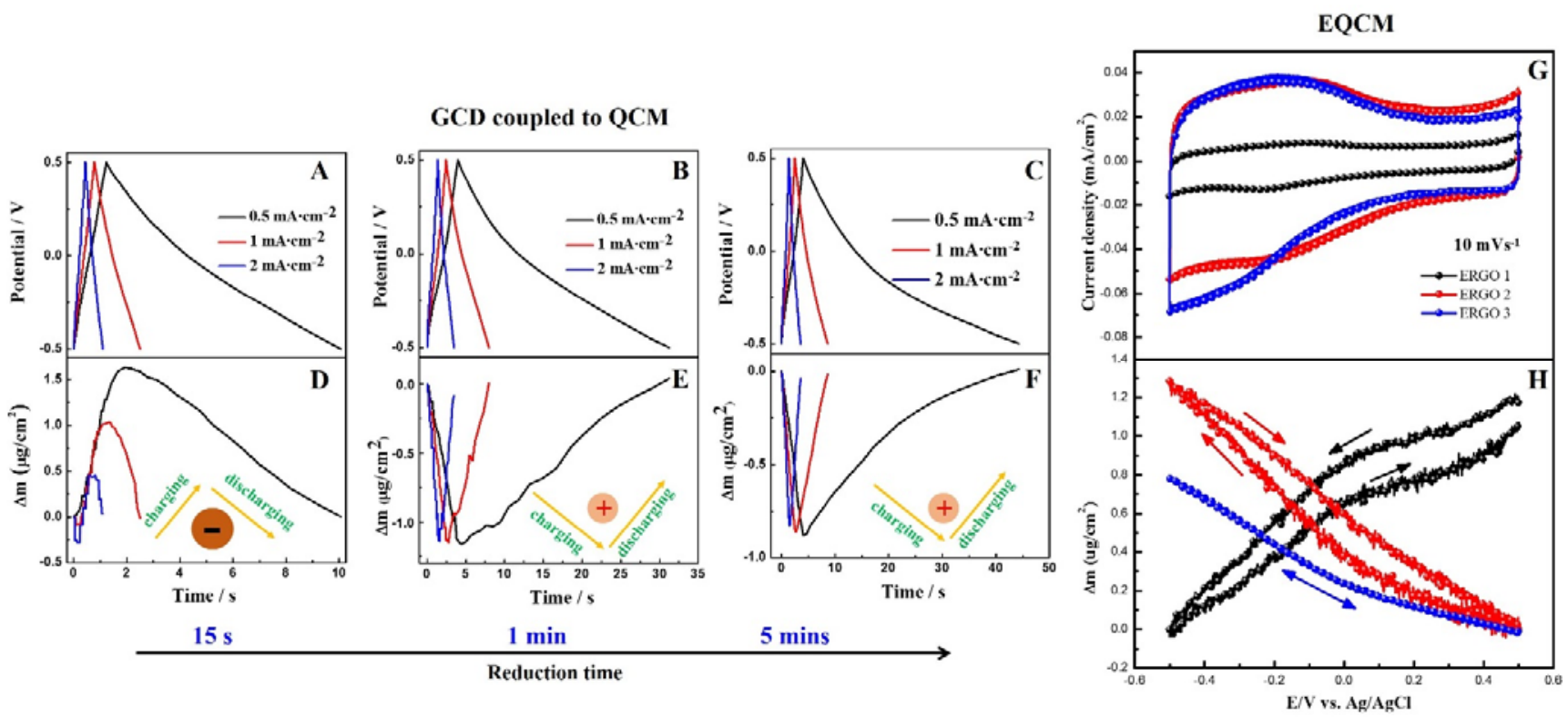

Figure 3. Galvanostatic charge-discharge of ERGO-1 (A, B), ERGO-2 (C, D) and ERGO-3 (E, F) at a current density of o.5, 1 and 2 $\mathrm{mA} \cdot \mathrm{cm}^{-2}$, respectively. Panels $(\mathrm{G})$ and $(\mathrm{H})$ are $\mathrm{CV}$ and mass change $(\Delta m)$ of ERGO electrodes obtained at $10 \mathrm{mV} \cdot \mathrm{s}^{-1}$.

the ionic transfer and thus allow much more species to be accommodated on the electrode during charge storage/delivery. And it is speculated that exchanged $\mathrm{H}_{2} \mathrm{O}$ molecules play an essential part in the variation of $G^{\prime \prime}$ by means of plasticizing effect ${ }^{46}$. These points will be scrutinized in the discussion part of the $A C$-EG data.

In addition to providing information on the electromechanical behavior, the electroacoustic studies on the ERGO layers may also permit the exploration of the validity of the gravimetric regime for the further analysis with the QCM and its satellite electrochemical methods. To estimate the influence of viscoelastic variations of ERGO electrode on its electrogravimetric performance, the experimental sensitivity factor of the quartz crystal resonator $\left(C_{f}=16.3 \times 10^{7}\right.$ $\mathrm{Hz} . \mathrm{g}^{-1} . \mathrm{cm}^{2}$ under the hypothesis of a sufficiently rigid electrode $^{28}$ ) was modified with the consideration of the rheological properties of the electrodes. The calculation was performed by Eq. 2 as previously reported ${ }^{29}$. Briefly, the film thickness was maintained constant $(\sim 146, \sim 122$ and $\sim 100 \mathrm{~nm}$ for ERGO-1, -2 and -3, respectively), $\rho_{f}^{\max }$ and $\rho_{f}^{\mathrm{min}}$ were confined between $\pm \rho_{f} \times 0.5 \%$ ( $\rho_{f}$ was obtained by the fitting procedure). The series frequency, $f_{s}^{V}$ , was calculated at each potential given that $G^{\prime}$ and $G^{\prime \prime}$ were constant upon a small potential perturbation.

$$
C_{f}^{\exp }=\frac{f_{s}^{V}\left(\rho_{f}^{\max }, h_{f}, G^{\prime}, G^{\prime \prime}\right)-f_{s}^{V}\left(\rho_{f}^{\min }, h_{f}, G^{\prime}, G^{\prime \prime}\right)}{\left(\rho_{f}^{\max }-\rho_{f}^{\min }\right) \times h_{f}}
$$

The resulting modified $C_{f}$ values for ERGO-1, -2 and -3 are $\sim 19.5 \times 10^{7}, \sim 18.7 \times 10^{7}$ and $\sim 17.8 \times 10^{7} \mathrm{~Hz} . \mathrm{g}^{-1} . \mathrm{cm}^{2}$, respectively. These modified values will be used in the following for the EQCM data analysis and for the fitting of the $A C$-EG data.

Cyclic electrogravimetric behaviour. The electrochemical behavior of ERGO electrodes was characterized by GCD and CV. The QCM was coupled with both measurements to track the simultaneous frequency shifts of the ERGO electrodes during cycling, which can be converted into mass responses. Typical GCD curves of three different ERGO electrodes obtained at a current density of $0.5,1$ and $2 \mathrm{~mA} \cdot \mathrm{s}^{-1}$ are shown in Figure $\mathbf{3} \mathbf{A}, \mathbf{B}$ and $\mathbf{C}$, while the corresponding mass changes are displayed in Figure $3 \mathbf{D}, \mathbf{E}$ and F. The charge/discharge curve of the ERGO-3 electrode presents a typical triangle shape with little deviation and is much prolonged over the ERGO-1 and -2 electrodes at various current densities, demonstrating that the capacitive behavior plays the major contribution in charge storage and that the specific capacitance of ERGO-3 electrode is significantly higher than those of ERGO-1 and $-2^{18}$. This reveals that significantly larger surface areas of the ERGO sheets become accessible for ions in charge storage with the progressive be removal of oxygen functionalities. Unlike the fairly clear evolution of potential responses, the mass responses of graphene oxides with different degree of reduction display a complex signature. As for ERGO-1, the mass-time response (Figure $3 \mathbf{D}$ ) proceeds in a synchronously positive-going pace with potential response, i.e., mass increase/decrease during charging/discharging, which is characteristic of anion participation in charge compensation process. By contrast, in the case of ERGO-2 and -3 , the mass response (Figure $3 \mathbf{E}$ and $\mathbf{F}$ ) shows a reverse triangular shape, evidencing a transition in the charge storage process, specifically from anions to cations transfer.

To confirm this "anion-to-cation" preference of the ERGO electrodes in electrochemical performance upon a progressive removal of oxygen functionalities, the EQCM was also performed. Figure $3 \mathbf{G}$ and Figure SI-2 (a-c) show approximately rectangular shaped $\mathrm{CV}$ responses with the 
slight contribution of broad redox peaks in the potential range of -0.5-0.2 V vs. $\mathrm{Ag} / \mathrm{AgCl}$, indicating that the charge storage corresponds mostly to a typical EDLC behavior. The minor deviations of an ideal rectangular response are due to the additional pseudocapacitances, which stem from the electrochemical reactions of remaining oxygen functionalities on ERGO layers at the electrode/electrolyte interface, e.g., $>\mathrm{C}-\mathrm{OH} \Leftrightarrow>\mathrm{C}=\mathrm{O}+\mathrm{H}^{+}+e^{-} 47,48$. In general, the specific capacitance $\left(C_{s}\right)$ of the electrode is proportional to its $\mathrm{CV}$ area ${ }^{49}$. Upon electrochemical reduction, the $C_{s}$ gradually increases. If the three ERGO (1-3) electrodes characterized at $10 \mathrm{mV} \cdot \mathrm{s}^{-1}$ are taken as an example (Figure ${ }_{3} \mathbf{G}$ ), an increase in the $C_{s}$ values is observed with the progressive removal of oxygen functional groups, as evidenced by the enlarged $\mathrm{CV}$ area. The maximum $C_{s}$ of $\sim 160.8 \mathrm{~F}^{\circ} \cdot \mathrm{g}^{-1}$ was obtained at a scan rate of $10 \mathrm{mV} \cdot \mathrm{s}^{-1}$ for the ERGO-3 electrode (Table SI-1), which exhibits a promising charge storage capability ${ }^{2,37}$.

Based on the data provided by GCD and CV coupled with QCM (Figure 3), anions seem to dominate in charge balance for ERGO-based electrodes with relatively high content of oxygen functionalities while cations' contribution is progressively enhanced with the decrease of oxygen functionalities on its layers. Therefore, it is likely to modify the microstructures of GO-based electrodes by means of regulating the content of oxygen functionalities on its layers, which can affect not only the electrochemical performances but also the nature of the ionic species participating in this process. However, the GCD and CV alone are not qualified to separate each species contribution and to provide their interfacial transfer kinetics ${ }^{28,50}$. For the deconvolution of the global electrogravimetric response into gravimetric and temporal components, the $A C$-EG was suggested as a complementary tool to the EQCM.

Ac-electrogravimetric investigations. $A C$-EG measurements were carried out at various states-of-polarization from $-0.4 \mathrm{~V}$ to $0.4 \mathrm{~V}$ vs. $\mathrm{Ag} / \mathrm{AgCl}$ with an interval of $100 \mathrm{mV}$. The electrochemical impedance, $\frac{\Delta E}{\Delta I}(\omega)$ and charge/potential transfer function (TF), $\frac{\Delta q}{\Delta E}(\omega)$ (Figure SI-3 and 4) of ERGO electrodes at selective potentials of $\pm 0.2 \mathrm{~V}$ vs. $\mathrm{Ag} / \mathrm{AgCl}$ are firstly compared, which can only reveal the contribution of charged species participating in the charge compensation process. These two potentials were shown herein as representatives of cathodic and anodic polarization states. The electrochemical impedance, (Figure SI-3) at low frequencies exhibits a slightly distorted straight line implying a multi-ion contribution to the charge compensation process. The experimental data of impedance and charge/potential TF (Figure SI-3 and SI-4) were fitted using theoretical functions in Eqs. 3 and 4 :

$$
\begin{aligned}
& \frac{\Delta E}{\Delta I}(\omega)=\left(j \omega d_{f} F \sum_{i} \frac{G_{i}}{j \omega d_{f}+K_{i}}\right)^{-1} \text { (i: ions) } \\
& \frac{\Delta q}{\Delta E}(\omega)=F d_{f} \sum_{i} \frac{G_{i}}{j \omega d_{f}+K_{i}} \text { (i: ions) }
\end{aligned}
$$

where $K_{i}$ represents the kinetics rate of transfer whereas $G_{i}$ describes the level of difficulty for each species transferred at the electrode/electrolyte interface. This fitting process revealed the involvement of three ionic species which resulted in a good agreement between the experimental and the theoretical curves (Figure 4 , Figure $\boldsymbol{S}_{\mathbf{3}}$ and $\mathbf{S}_{\mathbf{4}}$ ). Two parameters for each ionic species, $K_{i}$ and $G_{i}$, could be obtained through this fitting process and are listed in Table SI-2.

The charge/potential TF of the ERGO-1 exhibits a relatively small suppressed loop (or a composition of several loops) at both potentials (Figure ${ }_{4} \mathbf{C}$ and $\mathbf{D}$ ), demonstrating the presence of more than one charged species with similar time constant. In the case of ERGO-2, the $\frac{\Delta q}{\Delta E}(\omega)$ $\mathrm{TF}$ is distinguished by an enhancement of the loop diameter. After a further electrochemical reduction, ERGO-3 exhibits even a larger $\frac{\Delta q}{\Delta E}(\omega)$ TF loop at -0.2V with an upward tail at low frequency (LF). This suggests that at LF, another species may participate in charge balance and this species possesses a significantly different time constant to appear as a beginning of a separate loop. The impedance and charge/potential TF provide the contribution of charged species but do not permit their identification. Thus, $\frac{\Delta m}{\Delta E}(\omega)$ TFs were explored for tracking the flux of both charged and neutral species with their identification as well as for their kinetics of interfacial transfer (Figure $\mathbf{S}_{\mathbf{5}}$ ). Two parameters for each ionic species, $K_{i}$ and $G_{i}$ previously
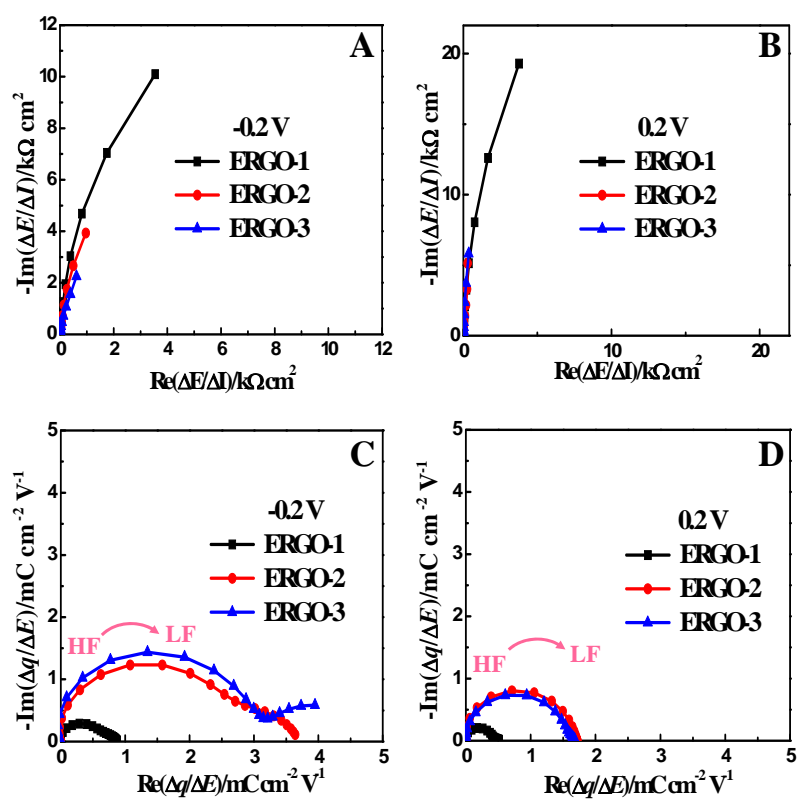

Figure 4. Theoretical curves of electrochemical impedance, $\Delta E / \Delta I(\omega)(\mathrm{A}, \mathrm{B})$ and the charge/potential transfer function, $\Delta q / \Delta E(\omega)(\mathrm{C}, \mathrm{D})$ of ERGO electrodes at $\pm 0.2 \mathrm{~V}$. For experimental curves, see Figure SI-3 and -4. 
obtained from impedance and charge/potential TF were used in the fitting process of the $\frac{\Delta m}{\Delta E}(\omega) \mathrm{TF}$, using Eq. 5: $\frac{\Delta m}{\Delta E}(\omega)=-d_{f} \sum_{i} M_{i} \frac{G_{i}}{j \omega d_{f}+K_{i}} \quad$ (i: ions and neutral species) (5)

The additional parameters, $K_{i}$ and $G_{i}$ for the solvent molecules and the molar mass $\left(M_{i}\right)$ of each species were estimated (listed in Table $\mathbf{S} 2$ at selective potentials of $\pm 0.2 \mathrm{~V}$ vs. $\mathrm{Ag} / \mathrm{AgCl})$.

Figure 5 depicts the evolution of $\frac{\Delta m}{\Delta E}(\omega)$ response of ERGO electrodes at $\pm 0.2 \mathrm{~V}$ vs. $\mathrm{Ag} / \mathrm{AgCl}$ with progressive removal of oxygen functionalities. Four different species, i.e., hydrated cations $\left(\mathrm{Na}^{+} \cdot \mathrm{nH}_{2} \mathrm{O}, \mathrm{n}=1\right.$ or 2$)$, cations $\left(\mathrm{Na}^{+}\right), \mathrm{H}_{2} \mathrm{O}$ molecules and anions $\left(\mathrm{Cl}^{-}\right)$were identified which resulted in a good agreement between the experimental and the theoretical curves (Figure $\mathbf{5}$ and Figure $\mathbf{S}_{\mathbf{5}}$ ). As shown in Scheme SI-1, the $\frac{\Delta m}{\Delta E}(\omega)$ response in the first quadrant is characteristic of an anion contribution, while a cation contribution appears in the third quadrant ${ }^{51}$. For ERGO-1, at both potentials, relatively small amount of hydrated cations $\left(\mathrm{Na}^{+} \cdot 2 \mathrm{H}_{2} \mathrm{O}\right)$ were identified at high frequencies (HF). They were followed by a significant contribution of anions $\left(\mathrm{Cl}^{-}\right)$and $\mathrm{H}_{2} \mathrm{O}$ molecules with the same flux direction with anions at high-intermediate and intermediate-low frequencies, respectively. Lastly, cations without hydration shell $\left(\mathrm{Na}^{+}\right)$were observed at very low frequencies with a minute contribution. Besides, no significant differences were observed between $-0.2 \mathrm{~V}$ and $0.2 \mathrm{~V}$. This illustrates a persistent electrochemical performance of ERGO-1 electrode with anions playing a major part in the charge compensation (Figure $5 \mathbf{A}$ and $\mathbf{B}$ ). On the contrary, the electrogravimetric response of ERGO-2 electrode presents a more obvious potential-dependent behavior. Specifically, at -0.2 $\mathrm{V}$, a fairly good agreement between the experimental and theoretical $\frac{\Delta m}{\Delta E}(\omega)$ data was achieved by considering the contribution of anions $\left(\mathrm{Cl}^{-}\right)$at low frequency (Figure $5 \mathbf{A}$ and $\mathrm{SI}_{-5} \mathrm{C}$ ). However, at $0.2 \mathrm{~V}$, this contribution disappeared and replaced by the extension of the contribution from $\mathrm{H}_{2} \mathrm{O}$ molecules with the same flux direction as cations (Figure $\left.{ }_{5} \mathrm{~B}\right)$.

ERGO-3 electrode presents one big loop of in the electrogravimetric TF in the third quadrant at high and intermediate frequencies, and a small one in the second quadrant at lower frequencies, independently of the applied potential (Figure $\mathbf{5}$ and $\mathbf{S}_{\mathbf{5}}$ ). It suggests a more homogeneous nanostructure of reduced graphene layers in ERGO-3 electrode with cations as a major charge carrier for charge balance in the potential range studied (-0.4 to $0.4 \mathrm{~V}$ ). Herein, it is worth mentioning that a drastic decrease of the loop diameter in the third quadrant was observed when the measurement potential was changed from -0.2 V to $0.2 \mathrm{~V}$ for ERGO-2 and -3 electrodes, implying a decrease of the cation/free solvent contributions.
It is interesting to mention that hydrated cation $\left(\mathrm{Na}^{+} \cdot \mathrm{nH}_{2} \mathrm{O}, \mathrm{n}=1\right.$ or 2$)$ transfers at the electrode/electrolyte interface with a higher kinetics than dehydrated one $\left(\mathrm{Na}^{+}\right)$, as shown in Figure 5. This can be explained by putting forward the existence of shallow electroactive sites close to the edge of ERGO layers and deep electroactive sites located in the inner of ERGO layers with higher activation energy $\left(E_{a}\right)$ for ion adsorption, which has been demonstrated in another $2 \mathrm{D}$ material, $\mathrm{Ti}_{3} \mathrm{C}_{2} \mathrm{~T}_{\mathrm{x}}$ MXenes $^{24}$. The shallow electroactive sites are quite accessible for hydrated cation adsorption due to lower $E_{a}$ for ion adsorption, leading to high transfer kinetics. However, deep electroactive sites are exclusively approachable for cations only after removing their hydration shell, which requires much more energy and results in lower transfer kinetics of $\mathrm{Na}^{+}$.

It has been demonstrated that during charge-discharge cycles, the exchange of ions between electrode and electrolyte is often accompanied with free solvent flux to satisfy the dimensional confinement of the electrode materials ${ }^{30}$. Moreover, the solvent exchanged often plays an important role in electrode's viscoelasticity through plasticizing effect ${ }^{45}$. For ERGO electrodes, exchanged free $\mathrm{H}_{2} \mathrm{O}$ and global $\mathrm{H}_{2} \mathrm{O}$ were calculated using Eqs. 6 and 7, where global $\mathrm{H}_{2} \mathrm{O}$ represents the sum of free $\mathrm{H}_{2} \mathrm{O}$ and the $\mathrm{H}_{2} \mathrm{O}$ molecules within the hydration shell of cations:
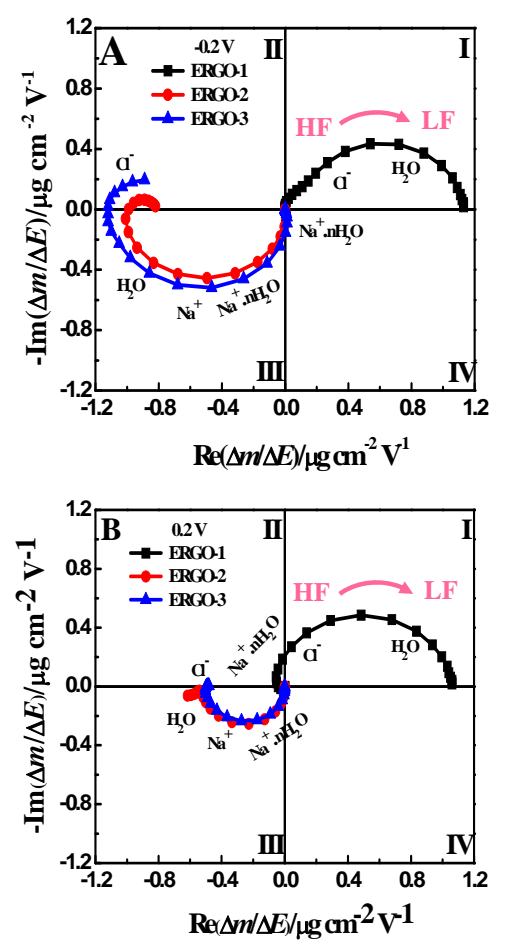

Figure 5. Theoretical curves of $\Delta m / \Delta E(\omega)$ transfer functions for ERGO electrodes at $-0.2 \mathrm{~V}$ and $0.2 \mathrm{~V}(\mathrm{a}, \mathrm{b})$. The details for fitting are shown in Figure $\mathrm{S}_{5}$. (HF: high frequency and LF: low frequency). 


$$
\begin{aligned}
& \mathrm{H}_{2} \mathrm{O}(\text { free })=\left|\frac{-G_{\mathrm{H}_{2} \mathrm{O}}}{K_{\mathrm{H}_{2} \mathrm{O}}}\right| \\
& \mathrm{H}_{2} \mathrm{O}(\text { global })=\left|\frac{-G_{\mathrm{H}_{2} \mathrm{O}}}{K_{\mathrm{H}_{2} \mathrm{O}}}\right|+x\left|\frac{-G_{\mathrm{Na}^{+}+\times \mathrm{H}_{2} \mathrm{O}}}{K_{\mathrm{Na}^{+}+\times \mathrm{H}_{2} \mathrm{O}}}\right|
\end{aligned}
$$

As shown in Figure S6-A and B, the increased $\mathrm{H}_{2} \mathrm{O}$ exchanged between electrode/electrolyte interface in ERGO1 from cathodic to anodic potentials (except $-0.4 \mathrm{~V}$ ) brings about progressively stronger plasticizing effect, contributing to the decrease of electrode's viscosity, i.e., lower $G^{\prime \prime}$ (Figure 2C). Contrarily, in ERGO-2 and ERGO-3, less $\mathrm{H}_{2} \mathrm{O}$ exchanged from cathodic to anodic potentials which may account for a higher $G^{\prime \prime}$ (Figure $\mathbf{2} \mathbf{C}$ ). Briefly, the viscoelasticity of the ERGO electrodes can be tuned through adjusting the content of the hetero-oxygen groups on its layers. More flexibility and less viscosity is expected during the process of removing oxygen functional groups.

\section{CONCLUSIONS}

The present study exhibited the significance of an integrated methodology of electroacoustic, EQCM and AC-EG measurements to probe the viscoelastic and electrochemical evolution of ERGO electrodes with different content of oxygen functionalities. During electrochemical cycling, two important parameters for describing the viscoelasticity of thin film ERGO electrodes, i.e., the storage $\left(G^{\prime}\right)$ and loss moduli ( $G$ ") are estimated. The viscoelastic parameters obtained from the electroacoustic admittance were further utilized to estimate the deviation of the sensitivity coefficients of the quartz crystal microbalance when it is not under the gravimetric regime and thus provided the differentiation of the effect of the mechanical property changes from purely gravimetric responses.

This combined research methodology can also be employed to obtain insights into the electro(chemical and mechanical) behavior for other $2 \mathrm{D}$ materials and might pave the way to understand the charge storage mechanism, facilitating the fabrication of highly efficient SC electrodes with ion-selective properties and superior long-term performances.

ASSOCIATED CONTENT: Supporting information available: Theoretical considerations for electroacoustic impedance and $A C$-EG methods providing further experimental data, fitting procedure details and the models used in the data analysis.

\section{AUTHOR INFORMATION}

Corresponding Authors: Ozlem Sel (ozlem.sel@upmc.fr) Hubert Perrot (hubert.perrot@upmc.fr)

ACKNOWLEDGEMENTS: Wanli Gao acknowledges China Scholarship Council - SU scholarship. We thank Ms. F. Pillier and S. Delbrel for the FEG-SEM, and Mr. C. Bazin for XRD.

\section{REFERENCES}

(1) Miller, J. R.; Simon, P., Electrochemical capacitors for energy management. Science 2008, 321 (5889), 651-652.

(2) Stoller, M. D.; Park, S. J.; Zhu, Y. W.; An, J. H.; Ruoff, R. S., Graphene-based ultracapacitors. Nano Lett. 2008, 8 (10), 34983502.

(3) Gogotsi, Y.; Simon, P., True performance metrics in electrochemical energy storage. Science 2011, 334 (6058), 917-918.

(4) Zhu, Y.; Murali, S.; Stoller, M. D.; Ganesh, K. J.; Cai, W.; Ferreira, P. J.; Pirkle, A.; Wallace, R. M.; Cychosz, K. A.; Thommes, M.; Su, D.; Stach, E. A.; Ruoff, R. S., Carbon-based supercapacitors produced by activation of graphene. Science 2011, 332 (6037), 15371541 .

(5) Lei, Z.; Christov, N.; Zhao, X. S., Intercalation of mesoporous carbon spheres between reduced graphene oxide sheets for preparing high-rate supercapacitor electrodes. Energy Environ. Sci. 2011, 4 (5), 1866-1873.

(6) Chen, L.; Zhang, X.; Liang, H.; Kong, M.; Guan, Q.; Chen, P.; $\mathrm{Wu}, \mathrm{Z}$.; Yu, S., Synthesis of nitrogen-doped porous carbon nanofibers as an efficient electrode material for supercapacitors. ACS Nano 2012, 6 (8), 7092-7102.

(7) Largeot, C.; Portet, C.; Chmiola, J.; Taberna, P.-L.; Gogotsi, Y.; Simon, P., Relation between the Ion Size and Pore Size for an Electric Double-Layer Capacitor. J. Am. Chem. Soc. 20o8, 130 (9), 27302731.

(8) Chmiola, J.; Yushin, G.; Gogotsi, Y.; Portet, C.; Simon, P.; Taberna, P. L., Anomalous increase in carbon capacitance at pore sizes less than 1 nanometer. Science 20o6, 313 (5794), 1760-1763.

(9) Salanne, M.; Rotenberg, B.; Naoi, K.; Kaneko, K.; Taberna, P. L.; Grey, C. P.; Dunn, B.; Simon, P., Efficient storage mechanisms for building better supercapacitors. Nat. Energy 2016, 1 (6), 16070. (10) Zhai, Y.; Dou, Y.; Zhao, D.; Fulvio, P. F.; Mayes, R. T.; Dai, S., Carbon materials for chemical capacitive energy storage. Adv. Mater. 2011, 23 (42), 4828-4850.

(11) El-Kady, M. F.; Strong, V.; Dubin, S.; Kaner, R. B., Laser scribing of high-performance and flexible graphene-based electrochemical capacitors. Science 2012, 335 (6074), 1326-1330.

(12) Wang, M.; Duong le, D.; Mai, N. T.; Kim, S.; Kim, Y.; Seo, H.; Kim, Y. C.; Jang, W.; Lee, Y.; Suhr, J.; Nam, J. D., All-solid-state reduced graphene oxide supercapacitor with large volumetric capacitance and ultralong stability prepared by electrophoretic deposition method. ACS Appl. Mater. Interfaces 2015, 7 (2), 1348-1354. (13) Wang, S.; Liu, N.; Su, J.; Li, L.; Long, F.; Zou, Z.; Jiang, X.; Gao, Y., Highly stretchable and self-healable supercapacitor with reduced graphene oxide based fiber springs. ACS Nano 2017, 11 (2), 2066-2074.

(14) Xu, J.; Tan, Z.; Zeng, W.; Chen, G.; Wu, S.; Zhao, Y.; Ni, K.; Tao, Z.; Ikram, M.; Ji, H.; Zhu, Y., A hierarchical carbon derived from sponge-templated activation of graphene oxide for high-performance supercapacitor electrodes. Adv. Mater. 2016, 28 (26), 5222-5228.

(15) Yang, X.; Zhu, J.; Qiu, L.; Li, D., Bioinspired effective prevention of restacking in multilayered graphene films: towards the next generation of high-performance supercapacitors. Adv. Mater. 2011, 23 (25), 2833-2838.

(16) Raccichini, R.; Varzi, A.; Passerini, S.; Scrosati, B., The role of graphene for electrochemical energy storage. Nat. Mater. 2015, 14 (3), 271-279.

(17) X-Y Peng; Liu, X.-X.; Diamond, D.; Lau, K. T., Synthesis of electrochemically-reduced graphene oxide film with controllable size and thickness and its use in supercapacitor. Carbon 2011, 49, 3488-3496.

(18) Xu, B.; Yue, S.; Sui, Z.; Zhang, X.; Hou, S.; Cao, G.; Yang, Y., What is the choice for supercapacitors: graphene or graphene oxide? Energy Environ. Sci. 2011, 4 (8), 2826. 
(19) Raymundo-Piñero, E.; Cadek, M.; Béguin, F., Tuning Carbon Materials for Supercapacitors by Direct Pyrolysis of Seaweeds. Adv. Funct. Mater. 2009, 19 (7), 1032-1039.

(20) Du, Q.; Zheng, M.; Zhang, L.; Wang, Y.; Chen, J.; Xue, L.; Dai, W.; Ji, G.; Cao, J., Preparation of functionalized graphene sheets by a low-temperature thermal exfoliation approach and their electrochemical supercapacitive behaviors. Electrochim. Acta 2010, 55 (12), 3897-3903.

(21) Yan, J.; Wang, Q.; Wei, T.; Fan, Z. J., Recent advances in design and fabrication of electrochemical supercapacitors with high energy densities. Adv. Energy Mater. 2014, 4 (4), 1300816.

(22) Dreyer, D. R.; Park, S.; Bielawski, C. W.; Ruoff, R. S., The chemistry of graphene oxide. Chem. Soc. Rev. 2010, 39 (1), 228-240. (23) Shpigel, N.; Levi, M. D.; Sigalov, S.; Mathis, T. S.; Gogotsi, Y.; Aurbach, D., Direct assessment of nanoconfined water in $2 \mathrm{D}$ $\mathrm{Ti}_{3} \mathrm{C}_{2}$ electrode interspaces by a surface acoustic technique. J. Am. Chem. Soc. 2018, 140 (28), 8910-8917.

(24) Levi, M. D.; Lukatskaya, M. R.; Sigalov, S.; Beidaghi, M.; Shpigel, N.; Daikhin, L.; Aurbach, D.; Barsoum, M. W.; Gogotsi, Y., Solving the capacitive paradox of $2 \mathrm{D}$ MXene using electrochemical quartz-crystal admittance and in situ electronic conductance measurements. Adv. Energy Mater. 2015, 5 (1), 1400815.

(25) Levi, M. D.; Shpigel, N.; Sigalov, S.; Dargel, V.; Daikhin, L.; Aurbach, D., In situ porous structure characterization of electrodes for energy storage and conversion by EQCM-D: a review. Electrochim. Acta 2017, 232, 271-284.

(26) Shpigel, N.; Lukatskaya, M. R.; Sigalov, S.; Ren, C. E.; Nayak, P.; Levi, M. D.; Daikhin, L.; Aurbach, D.; Gogotsi, Y., In situ monitoring of gravimetric and viscoelastic changes in $2 \mathrm{D}$ intercalation electrodes. ACS Energy Lett. 2017, 2 (6), 1407-1415.

(27) Goubaa, H.; Escobar-Teran, F.; Ressam, I.; Gao, W.; El Kadib, A.; Lucas, I. T.; Raihane, M.; Lahcini, M.; Perrot, H.; Sel, O., Dynamic resolution of ion transfer in electrochemically reduced graphene oxides revealed by electrogravimetric impedance. J. Phys. Chem. C 2017, 121 (17), 9370-9380.

(28) Escobar-Teran, F.; Arnau, A.; Garcia, J. V.; Jiménez, Y.; Perrot, H.; Sel, O., Gravimetric and dynamic deconvolution of global EQCM response of carbon nanotube based electrodes by Ac-electrogravimetry. Electrochem. Commun. 2016, 70, 73-77.

(29) García-Jareño, J. J.; Gabrielli, C.; Perrot, H., Validation of the mass response of a quartz crystal microbalance coated with Prussian Blue film for ac electrogravimetry. Electrochem. Commun. 2000, 2 (3), 195-200.

(30) Hillman, A. R.; Efimov, I.; Skompska, M., Dynamics of regioregular conducting polymer electrodes in response to electrochemical stimuli. Faraday Discuss. 2002, 121, 423-439.

(31) Koehler, S.; Bund, A.; Efimov, I., Shear moduli of anion and cation exchanging polypyrrole films. J. Electroanal. Chem. 2oo6, $589(1), 82-86$.

(32) Hummers, W. S. J.; Offeman, R. E., Preparation of graphitic oxide. J. Am. Chem. Soc. 1958, 8o (6), 1339-1339.

(33) Granstaff, V. E.; Martin, S. J., Characterization of a thicknessshear mode quartz resonator with multiple nonpiezoelectric layers. J. Appl. Phys. 1994, 75 (3), 1319-1329.

(34) Gao, W.; Sel, O.; Perrot, H., Electrochemical and viscoelastic evolution of dodecyl sulfate-doped polypyrrole films during electrochemical cycling. Electrochim. Acta 2017, 233, 262-273.

(35) Hook, F.; Kasemo, B. Variations in coupled water, viscoelastic properties, and film thickness of a Mefp-1 protein film during adsorption and cross-Linking: A quartz crystal microbalance with dissipation monitoring, ellipsometry, and surface plasmon resonance study. Anal. Chem. 2001, 73, 5796-5804.
(36) G. Sauerbrey, Verwendung von Schwingquarzen zur Wägung dünner Schichten und zur Mikrowägung. Zeitschrift für physik 1959, 155, 206-222.

(37) Shao, Y.; Wang, J.; Engelhard, M.; Wang, C.; Lin, Y., Facile and controllable electrochemical reduction of graphene oxide and its applications. J. Mater. Chem. 2010, 20 (4), 743-748.

(38) Pei, S.; Zhao, J.; Du, J.; Ren, W.; Cheng, H.-M., Direct reduction of graphene oxide films into highly conductive and flexible graphene films by hydrohalic acids. Carbon 2010, 48 (15), 44664474 .

(39) Shin, H. J.; Kim, K. K.; Benayad, A.; Yoon, S. M.; Park, H. K.; Jung, I. S.; Jin, M. H.; Jeong, H. K.; Kim, J. M.; Choi, J. Y.; Lee, Y. $\mathrm{H}$., Efficient reduction of graphite oxide by sodium borohydride and its effect on electrical conductance. Adv. Funct. Mater. 2009, 19 (12), 1987-1992.

(40) Chen, Y.; Zhang, X.; Zhang, D.; Yu, P.; Ma, Y., High performance supercapacitors based on reduced graphene oxide in aqueous and ionic liquid electrolytes. Carbon 2011, 49 (2), 573-580.

(41) Debiemme-Chouvy, C.; Thomas, B.; Lucas, I. T.; Tran, T. T. M.; Heintz, J. M.; Veillere, A.; Silvain, J. F., Facile and green reduction of graphene oxide by a reduced polyoxometalate and formation of a nanohybrid. Chem Plus Chem 2017, 82 (2), 186-189.

(42) Fan, X.; Peng, W.; Li, Y.; Li, X.; Wang, S.; Zhang, G.; Zhang, F., Deoxygenation of exfoliated graphite oxide under alkaline conditions: a green route to graphene preparation. Adv. Mater. 20o8, 20 (23), 4490-4493.

(43) Xu, Y; Sheng, K.; Li, C.; Shi, G., Self-assembled graphene hydrogel via a one-step hydrothermal process. ACS Nano 2010, 4 (7), 4324-4330.

(44) Dargel, V.; Jackel, N.; Shpigel, N.; Sigalov, S.; Levi, M. D.; Daikhin, L.; Presser, V.; Aurbach, D., In situ multilength-scale tracking of dimensional and viscoelastic changes in composite battery electrodes. ACS Appl. Mater. Interfaces 2017, 9 (33), 2766427675 .

(45) Mohamoud, M. A.; Hillman, A. R., The effect of anion identity on the viscoelastic properties of polyaniline films during electrochemical film deposition and redox cycling. Electrochim. Acta 2007, 53 (3), 1206-1216.

(46) Hillman, A. R.; Mohamoud, M. A.; Efimov, I., Time-temperature superposition and the controlling role of solvation in the viscoelastic properties of polyaniline thin films. Anal. Chem. 2o11, 83 (14), 5696-5707.

(47) Bao, Q.; Bao, S.; Li, C.; Qi, X.; Pan, C.; Zang, J.; Lu, Z.; Li, Y.; Tang, D.; Zhang, S.; Lian, K., Supercapacitance of solid carbon nanofibers made from ethanol flames. J. Phys. Chem. C 2008, 112, 3612-3618.

(48) Zhao, B.; Liu, P.; Jiang, Y.; Pan, D.; Tao, H.; Song, J.; Fang, T.; $\mathrm{Xu}, \mathrm{W}$., Supercapacitor performances of thermally reduced graphene oxide. J. Power Sources 2012, 198, 423-427.

(49) Yang, Q.; Bi, R.; Yung, K.-c.; Pecht, M., Electrochemically reduced graphene oxides/nanostructured iron oxides as binder-free electrodes for supercapacitors. Electrochim. Acta 2017, 231, 125134

(5o) Gabrielli, C.; Garcia-Jareño, J. J.; Perrot, H., Charge compensation process in polypyrrole studied by ac electrogravimetry. Electrochim. Acta 2001, 46 (26), 4095-4103.

(51) Gabrielli, C.; Garcia-Jareno, J. J.; Keddam, M.; Perrot, H.; Vicente, F., Ac-electrogravimetry study of electroactive thin films. II. Application to polypyrrole. J. Phys. Chem. B 2002, 106, 31923201. 
Electrochemical Reduction process

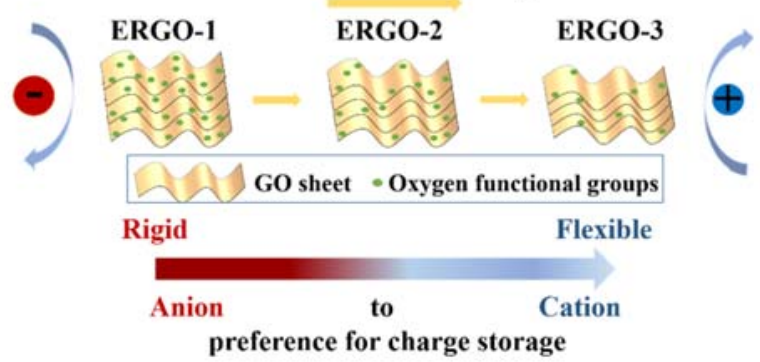

Revealed by an integrated QCM based methodology, progressive removal of oxygen-functionalities of ERGO induces evolution from "anion-to-cation preference" for charge storage/delivery, playing significant role in electrode's viscoelasticity. 